\title{
Estimation of the Bit Error Rate (BER) for Uplink Millimeter-Wave Line-of-Sight Communications
}

\author{
M. Comisso, F. Vatta, G. Buttazzoni, and F. Babich \\ Department of Engineering and Architecture, University of Trieste, Italy, \\ E-mail: $\{$ mcomisso, vatta, gbuttazzoni, babich\}@units.it
}

\begin{abstract}
This paper proposes a theoretical analysis for evaluating the statistical distribution of the bit error rate (BER) for a millimeter-wave uplink communication involving a mobile terminal (MT) and a base station (BS). The analysis is focused on the line-of-sight scenario, whose investigation provides useful insights on the maximum performance achievable by forthcoming fifth-generation (5G) wireless networks. The developed model accounts for path-loss attenuation and directional antenna gains assuming a noise-limited operating regime. The location of the MTs around the 5G BS is described by a Poisson point process, while the statistic of the BER is estimated by considering the phase-shift keying (PSK) and the quadrature amplitude modulation (QAM) schemes. The analytical results are finally exploited to discuss the dependence of the error rate from the cell radius and the modulation order in a mmWave context.
\end{abstract}

Index Terms-Millimeter-wave communication; line-of-sight; modulation; BER.

\section{INTRODUCTION}

The mature implementation of the long-term evolution (LTE) system has boosted the interest of commercial operators towards the fifth-generation $(5 \mathrm{G})$ cellular network, whose placement on the market is expected to be realized by 2020 [1]. The forthcoming 5G technology foresees a pervasive wireless scenario capable to support the most evolved Internet of Things (IoT) applications, from high-definition video streaming to intelligent metering, from automated health to infrastructure management, home automation and public transportation [2-4]. Beside the provisioning of more capacity with respect to LTE, $5 \mathrm{G}$ is expected to be characterized by a major virtualization of the core and edge network, an ultra-densification of the deployed devices, and a shift of the frequency spectrum towards the millimeter-wave (mmWave) domain. Just this latter feature represents a challenge for telecommunications' researchers in terms of design of radio transceivers and radiating structures. The reduced carrier wavelength, in fact, enables to package many antennas on a single device, so as to support high-gain directional links capable to compensate the significant attenuations that characterize the mmWave propagation environment. Thus, similarly to previously proposed extensions for personal and local area networks [5-8], also 5G will benefit from massive multipleinput multiple-output (MIMO) systems.

The theoretical characterization of the performance achievable by a mmWave link is still in progress, even if valuable

\footnotetext{
${ }^{1}$ This work is partly supported by the Italian Ministry of University and Research within project FRA 2018 (Univ. of Trieste, Italy) "UBER-5G: Cubesat 5G networks - Access layer analysis and antenna system development."
}

frameworks have been already published [9-15]. Most of these studies focus on the estimation of the coverage probability or on the transitional behavior from an interference-limited regime, in which the power incoming from the undesired sources prevails over the noise, to a noise-limited regime, in which, differently, the noise power prevails over the interference. Thus, currently, the main addressed issue concerns the distribution of the signal to interference plus noise ratio (SINR) or of the signal to noise ratio (SNR), depending on the operating regime. Few of the proposed works on mmWave cellular systems, however, discuss in detail the bit error rate (BER) resulting from the SINR/SNR distribution on the basis of the adopted modulation scheme, a topic that has been instead widely analyzed for conventional microwave ( $\mu$ Wave) networks [16-20].

This study addresses this latter aspect by presenting a theoretical analysis for estimating the BER of a mmWave uplink communication. To better focus the investigation on the achievable performance, a line-of-sight (LOS) scenario is modeled, where a mobile terminal (MT) communicates with a $5 \mathrm{G}$ base station (BS). The LOS scenario is selected for its particular interest, since the forthcoming $5 \mathrm{G}$ technology is expected to express its maximum potential specifically in LOS conditions, revealing the actual capacity outbreak of $5 \mathrm{G}$ with respect to the currently used $3 \mathrm{G} / 4 \mathrm{G}$ cellular systems [21], [22]. To properly address a realistic environment, the path-loss propagation model is derived in agreement with experimental data acquired by recent measurement campaigns at $28 \mathrm{GHz}$. Besides, the MTs are assumed distributed according to a Poisson point process (PPP), while the commonly employed phase-shift keying (PSK) and quadrature amplitude modulation (QAM) schemes are considered to determine the statistic of the BER. The conceived analysis is finally exploited to investigate the influence of the cell radius and of the modulation order on the mmWave link performance.

The paper is organized as follows. Section II introduces the system model. Section III presents the theoretical analysis. Section IV discusses the numerical results. Section V summarizes the main conclusions.

Notation. Throughout the paper the following notation is used: $\mathbb{R}_{>0}$ and $\mathbb{R}_{\geq 0}$ denote the sets of positive and nonnegative reals, respectively; $\mathbb{1}_{\mathbf{X}}(\mathbf{x})$ denotes the indicator function (i.e., $\mathbb{1}_{\mathbf{X}}(\mathbf{x})=1$ if $\mathbf{x} \in \mathbf{X}, \mathbb{1}_{\mathbf{X}}(\mathbf{x})=0$ if $\mathbf{x} \notin \mathbf{X}$ ); $W(x)$ denotes the Lambert W-function; $Q(x)$ denotes the $\mathrm{Q}$-function and $Q^{-1}(x)$ its inverse. 


\section{SySTEM MODEL}

Consider a two-dimensional mmWave cellular network in which a given BS communicates with a fixed MT. The positions of the MTs around the BS are described by a homogeneous PPP of intensity $\lambda$, and the BS serves the closest MT among those present in the corresponding cell [11]. Thus, according to the nearest neighbor statistic, the MT-BS distance is a random variable (r.v.) $R$ having cumulative distribution function (cdf) [23]:

$$
F_{R}(r)=\left[1-\exp \left(-\pi \lambda r^{2}\right)\right] \mathbb{1}_{\mathbb{R}_{\geq 0}}(r) .
$$

Recent measurement campaigns and detailed theoretical investigations have shown that path-loss and noise are two of the most relevant propagation phenomena that determine the link state of a mmWave communications [9], [21]. More precisely, three states are commonly taken into account: LOS, when the MT-BS link is characterized by optical visibility; NLOS, when the optical visibility is not present by anyway the communication is possible; and outage (OUT), when the pathloss is so high that the MT-BS link cannot be established [11]. Among these three possible situations, the most favorable one is the first, since in LOS conditions the mmWave technology provides its largest potential as compared to current 2-4G $\mu$ Wave systems. For this reason, the LOS case is analyzed in detail, with the aim of deriving an upper bound for the BER of a mmWave uplink communication. The presence of a direct LOS visibility is however a statistical event, whose occurrence depends on the MT-BS distance. In particular, assuming as negligible the probability of being in the OUT state (i.e., considering not too large cells), the probability of being a LOS state is given by [21]:

$$
p_{\mathrm{LOS}}(R)=\exp (-a R),
$$

where the LOS parameter $a$, which depends on the propagation environment and on the operating frequency, may be derived by fitting of measured data. An estimation of this parameter for the 28 and $73 \mathrm{GHz}$ channels has been derived in [21], where the value $a \cong 14.9 \mathrm{~mm}^{-1}$ is obtained. This enables to preliminarily quantify the probability of being in LOS conditions for different distances. In particular, for $R=10,50,100,150 \mathrm{~m}$, one obtains $p_{\text {LOS }}(R) \cong 0.86,0.47,0.23,0.11$, which reveal that, even in a mmWave environment, the LOS state, as expected, usually occurs at the lower distances, remaining however significantly possible at the larger ones.

According to the experimental characterization realized in [21], the omnidirectional path-loss attenuation is assumed to linearly depend on the logarithm of the distance, thus it is modeled by the function:

$$
\rho(R)=\frac{1}{\alpha R^{\beta}},
$$

where $\alpha$ denotes the floating intercept and $\beta$ represents the average path-loss exponent, which is again derived by performing a best-fit linear regression of empirical data. While the setting $\beta=2$ is typical for the LOS scenario regardless of the specific frequency adopted for the communication, the value of $\alpha$ is influenced by the selected band. For the two parameters $a$ and $\alpha$, the experimental estimations provided in
[21, Table I] will be adopted in this paper. Since both the BS and the MT are assumed fixed, mobility effects due to midand small-scale fading are not considered.

A relevant quantity that must be included when the performance of a mmWave communication has to be evaluated is the noise power. This quantity may be calculated at the receiving BS as [11]:

$$
\sigma=\mathcal{N}_{0} \cdot B \cdot \mathcal{F}
$$

where $\mathcal{N}_{0} \cong 3.98 \cdot 10^{-21} \mathrm{~W} / \mathrm{Hz}$ is the noise spectral density, $B$ is the receiver bandwidth, and $\mathcal{F}$ is its noise figure. Differently from conventional $\mu$ Wave networks, which usually operate in an interference-limited regime, it has been proved that mmWave communications may be properly modeled by pseudowired links as long as sufficiently directional antennas are adopted. This implies that, in the presence of high-gain radiating systems [24-26], not too dense mmWave networks operate in a noise-limited regime [9].

\section{ANALYSIS}

In agreement with the introduced scenario, this section evaluates the statistical BER performance for the monitored MT-BS mmWave uplink. This task is accomplished by first calculating the distribution of the power received at the BS, then that of the corresponding SNR, and finally the cdf of the resulting BER according to selected modulation scheme.

Recalling the formula for the LOS probability in (2) and the path-loss function in (3), one can express the fraction of the average power received by the BS from the target MT that is due to the sole LOS contribution through the r.v.:

$$
\begin{aligned}
P & =P_{\mathrm{T}} G_{\mathrm{T}} G_{\mathrm{R}} \cdot \rho(R) \cdot p_{\mathrm{LOS}}(R) \\
& =\frac{K}{R^{\beta}} \exp (-a R),
\end{aligned}
$$

where $P_{\mathrm{T}}$ is the transmission power, $G_{\mathrm{T}} / G_{\mathrm{R}}$ are the transmitting/receiving antenna power gains, and:

$$
K=\frac{P_{\mathrm{T}} G_{\mathrm{T}} G_{\mathrm{R}}}{\alpha} .
$$

The cdf $F_{P}(p)$ of $P$ can be derived by inverting (5) with respect to $R$ and then using (1) to account for the statistical location of the MT. This yields:

$$
\begin{aligned}
F_{P}(p) & =\operatorname{Pr}\{P \leq p\} \\
& =\operatorname{Pr}\left\{\frac{K}{R^{\beta}} \exp (-a R) \leq p\right\} \\
& =1-F_{R}\left\{\frac{\beta}{a} W\left[\left(\frac{\nu}{p}\right)\right]\right\} \\
& =\exp \left\{-\gamma W^{2}\left[\left(\frac{\nu}{p}\right)^{\frac{1}{\beta}}\right]\right\} \mathbb{1}_{\mathbb{R}_{>0}}(p),
\end{aligned}
$$

where $\gamma=\pi \lambda(\beta / a)^{2}$ and $\nu=K(a / \beta)^{\beta}$. Note that the inverse of (5), which cannot be described in closed-form by elementary functions, can be formally expressed through the Lambert W-function, which is increasingly monotone for nonnegative arguments [27]. Besides, by calculating the derivative of (7), 
one can directly obtain the probability density function (pdf) of the received power as:

$$
f_{P}(p)=\frac{2 \gamma W^{2}\left[\left(\frac{\nu}{p}\right)^{\frac{1}{\beta}}\right] \exp \left\{-\gamma W^{2}\left[\left(\frac{\nu}{p}\right)^{\frac{1}{\beta}}\right]\right\}}{\beta p\left\{1+W\left[\left(\frac{\nu}{p}\right)^{\frac{1}{\beta}}\right]\right\}} \mathbb{1}_{\mathbb{R}_{>0}}(p) .
$$

According to the assumption of noise-limited regime [11], the impact of the interference on the monitored MT-BS mmWave communication may be assumed negligible. This implies that the result of this communication depends on the sole noise, and in particular on the r.v. $\Psi$ describing the SNR, which is a function of $P$ and can be defined as:

$$
\Psi(P)=\frac{P}{\sigma},
$$

where $\sigma$ is given by (4). By remembering the rule for the scaling of r.v.s [28] and using (7), the cdf of $\Psi$ may be immediately evaluated as:

$$
\begin{aligned}
F_{\Psi}(\psi) & =F_{P}(\sigma \psi) \\
& =\exp \left\{-\gamma W^{2}\left[\left(\frac{\nu}{\sigma \psi}\right)^{\frac{1}{\beta}}\right]\right\} \mathbb{1}_{\mathbb{R}_{>0}}(\psi) .
\end{aligned}
$$

The final step necessary to evaluate the statistic of the BER requires to include the modulation employed for data transmission. To this aim, two widely adopted possibilities are considered: the $M$-PSK and the $M$-QAM schemes, in which the parameter $M$ identifies the modulation order. Since, in a noise-limited regime, the BER is related to the SNR, it can be described by a r.v. $\Upsilon$, which, for both schemes, may be suitably approximated by a function of $\Psi$ as [29], [30]:

$$
\Upsilon(\Psi) \cong \xi_{M} Q\left(\zeta_{M} \sqrt{\Psi}\right)
$$

where the constants $\xi_{M}$ and $\zeta_{M}$ depend on the specific modulation and are given by:

$$
\begin{gathered}
\xi_{M}=\frac{2}{\log _{2} M} \cdot \begin{cases}1 & M-\mathrm{PSK} \\
2\left(1-\frac{1}{\sqrt{M}}\right) & M-\mathrm{QAM}\end{cases} \\
\zeta_{M}=\sqrt{\log _{2} M} \cdot \begin{cases}\sqrt{2} \sin \left(\frac{\pi}{M}\right) & M-\mathrm{PSK} \\
\sqrt{\frac{3}{M-1}} & M-\mathrm{QAM}\end{cases}
\end{gathered}
$$

The cdf $F_{\Upsilon}(v)$ of $\Upsilon$ may be calculated from the inverse of (11) with respect to $\Psi$ and subsequently applying (10). Remembering that the Q-function is monotone decreasing, these two latter operations lead to:

$$
\begin{aligned}
F_{\Upsilon}(v) & =\operatorname{Pr}\{\Upsilon \leq v\} \\
& \cong \operatorname{Pr}\left\{\xi_{M} Q\left(\zeta_{M} \sqrt{\Psi}\right) \leq v\right\}
\end{aligned}
$$

\begin{tabular}{llll}
\hline$P_{\mathrm{T}}$ & $100 \mathrm{~mW}$ & $\alpha$ & $61.4 \mathrm{~dB}$ \\
\hline$G_{\mathrm{T}}$ & $10 \mathrm{~dB}$ & $\beta$ & 2 \\
\hline$G_{\mathrm{R}}$ & $10 \mathrm{~dB}$ & $a$ & $14.9 \mathrm{~mm}^{-1}$ \\
\hline$B$ & $1 \mathrm{GHz}$ & $\mathcal{F}$ & $10 \mathrm{~dB}$ \\
\hline \multicolumn{4}{c}{ TABLE I }
\end{tabular}

ADOPTED PARAMETERS [21].

$$
\begin{aligned}
& =1-F_{\Psi}\left\{\left[\frac{1}{\zeta_{M}} Q^{-1}\left(\frac{v}{\xi_{M}}\right)\right]^{2}\right\} \\
& =\{1-\exp [-\gamma H(v)]\} \mathbb{1}_{\mathbb{R}_{>0}}(v),
\end{aligned}
$$

where:

$$
H(v)=W^{2}\left\{\left(\frac{\nu}{\sigma}\right)^{\frac{1}{\beta}}\left[\frac{1}{\zeta_{M}} Q^{-1}\left(\frac{v}{\xi_{M}}\right)\right]^{-\frac{2}{\beta}}\right\} .
$$

The final statistic in (13) represents the probability that the BER lies below a given threshold $v$ when the MT-BS mmWave communication link operates in LOS conditions. The accuracy of this result is checked in the next section by comparing the theoretical analysis with numerical simulations.

\section{RESULTS}

The results are obtained using the parameters in Table I, which are referred to the measurements in the $28 \mathrm{GHz}$ band carried out in [21]. The values provided by the analysis are validated by independent Monte Carlo simulations. In the presented figures, the theoretical curves will be identified by lines, while those derived from simulations will be denoted by markers. All results are evaluated using Matlab and running $S=100000$ realizations for each point of a simulation curve.

The first set results is shown in Fig. 1, which reports the cdf of the power received by the 5G BS (Fig. 1(a)), and the corresponding pdf (Fig. 1(b)), for different values of the cell radius $\varrho$. Note that this latter parameter determines the intensity of the PPP describing the location of the MTs, since $\lambda$ and $\varrho$ are related by [11]:

$$
\lambda=\frac{1}{\pi \varrho^{2}} .
$$

Thus, the lower the cell radius, the higher the intensity of the sources and, in turn, the closer the transmitting MT to the receiving BS. As it may be observed from Fig. 1(a), this implies that, for a given $p$ value, the probability that the received power be lower than $p$ increases with the increase of $\varrho$. Note that this behavior holds until the network operates in a noise-limite regime, that is, until the transmitting/receiving antenna radiation patterns are sufficiently directional to completely filter, in the spatial domain, the interference of the other MTs. In the presence of very high $\lambda$ values, instead, this latter assumption cannot be considered acceptable, since, as shown in [31], ultra-dense scenarios tend to operate in an interference-limite regime. As a final remark concerning Fig. 1(b), it has been verified that the maximum of each pdf corresponds to the inflexion of the relative cdf in Fig. 1(a). This does not appear immediately because of the usage, for 


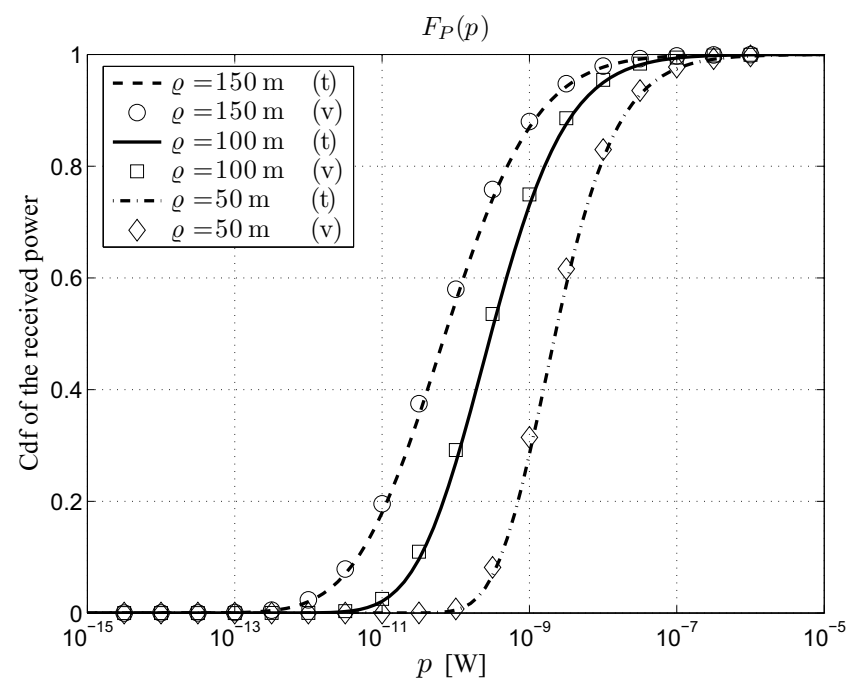

(a)

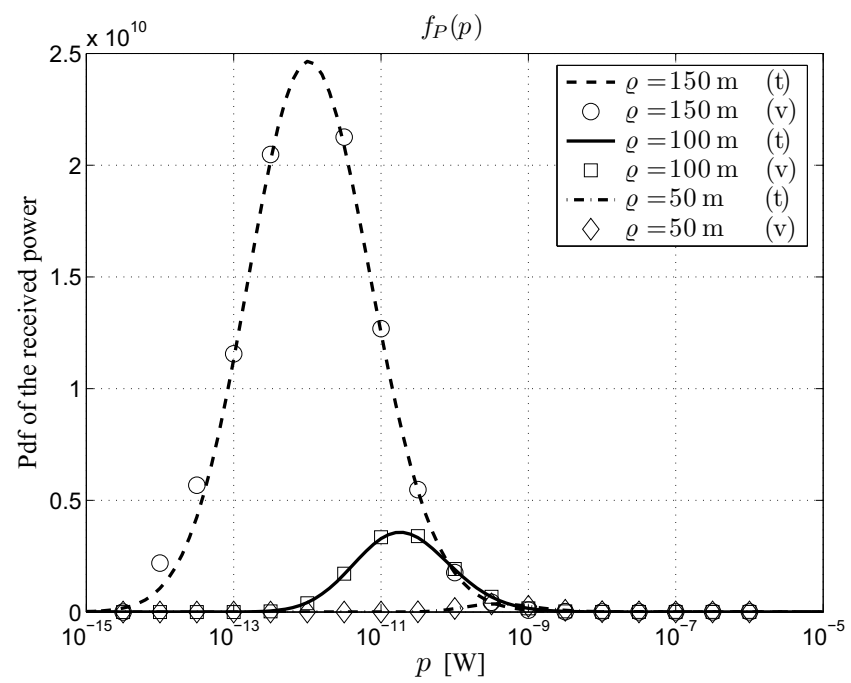

(b)

Fig. 1. Statistics of the power received by the BS for different values of the cell radius: (a) cdf, (b) pdf (t: theory, v: Monte Carlo validation).

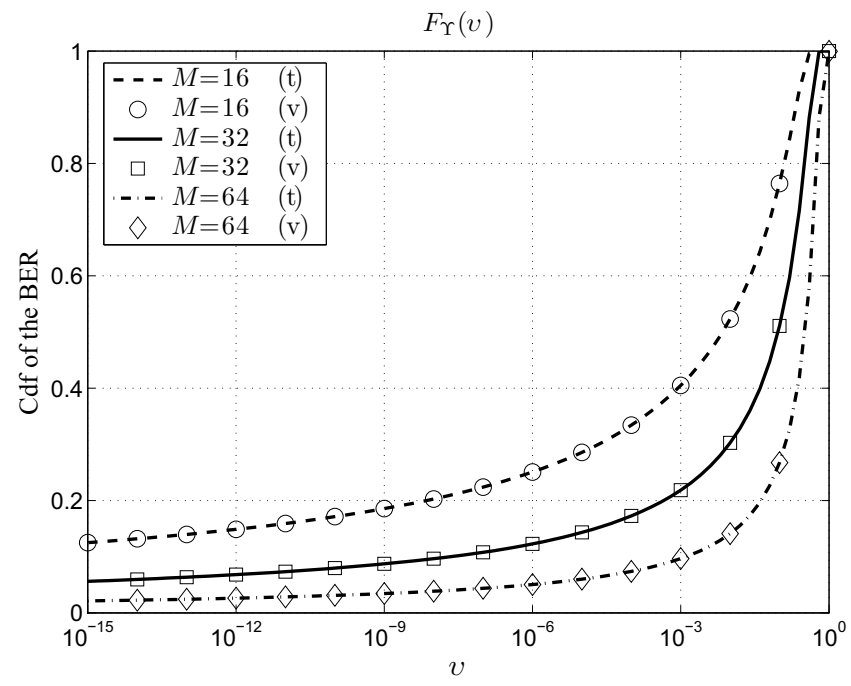

(a)

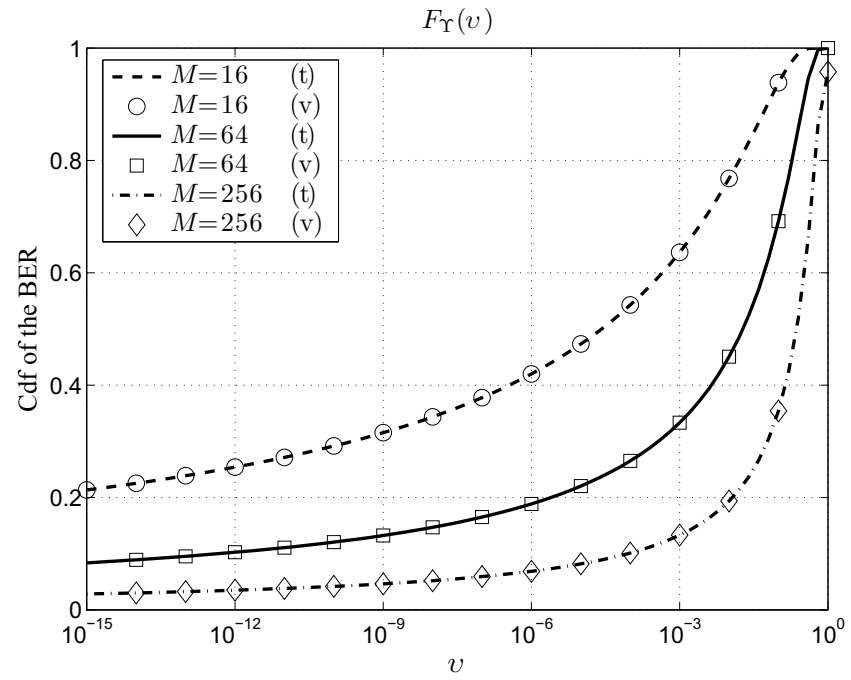

(b)

Fig. 2. Cdf of the BER for $\varrho=50 \mathrm{~m}$ and different values of the modulation order: (a) $M$-PSK, (b) $M$-QAM (t: theory, v: Monte Carlo validation).

readability reasons, of a log-scale for the $p$-axis. Besides, the area under each curve has been verified to be equal to one, as it must be for any pdf to satisfy the normalization condition. In this regard, the lower the maximum of a pdf, the longer the tail for large $p$ values, whose contribution to the overall area under the pdf becomes hence more relevant.

The second set of results is reported in Fig. 2, which shows the cdf of the BER obtained considering cells of radius $\varrho=50 \mathrm{~m}$ and different modulation schemes. More precisely, Fig. 2(a) refers to the $M$-PSK modulation, while Fig. 2(b) to the $M$-QAM one. As expected, for a given scheme, the increase of the modulation order reduces the probability of obtaining a BER lower than a given value, since, for a constant transmission power, the symbols of the constellation get closer with the increase of $M$. Besides, the $M$-QAM scheme, which operates on both the amplitude and the phase of the signal, provides better statistics as compared to the $M$-PSK one, which instead operates only on the phase of the signal. This enables the adoption of $M$-QAM also for high $M$ values when significant data rates are required and satisfactory channel conditions are present.

Similar observations may be formulated considering the last set of results (Fig. 3), which show the cdf of the BER for $\varrho=$ $100 \mathrm{~m}$ when the $M$-PSK (Fig. 3(a)) and the $M$-QAM schemes (Fig. 3(b)) are adopted. The main difference that can be noticed with respect to Fig. 2 concerns the lower performance due to the larger cell radius, which implies statistically farther MTs. In fact, comparing, for a given modulation and a given $M$ value, two corresponding curves in Figs. 2 and 3, the second one is characterized by a reduced BER. In general, 


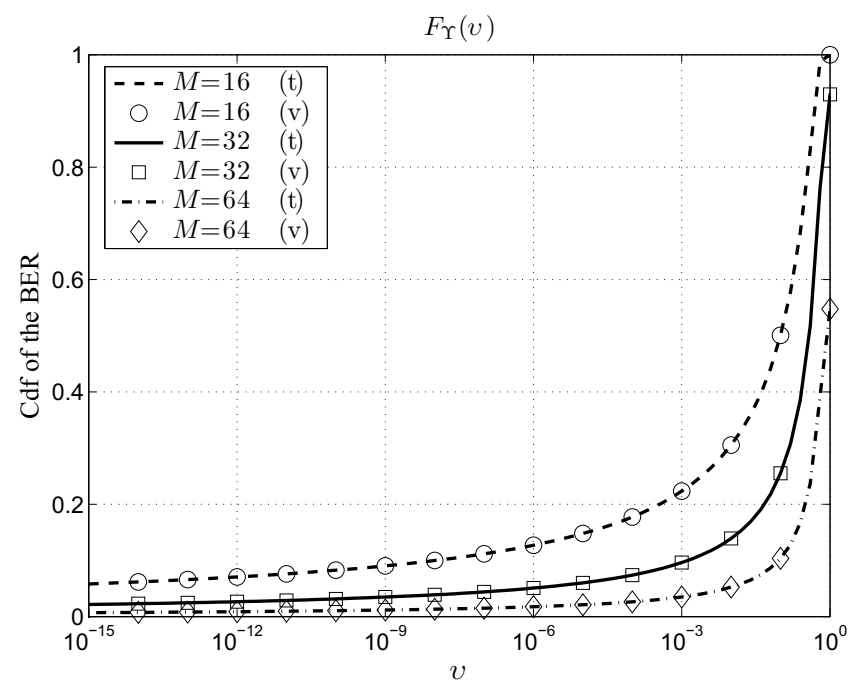

(a)

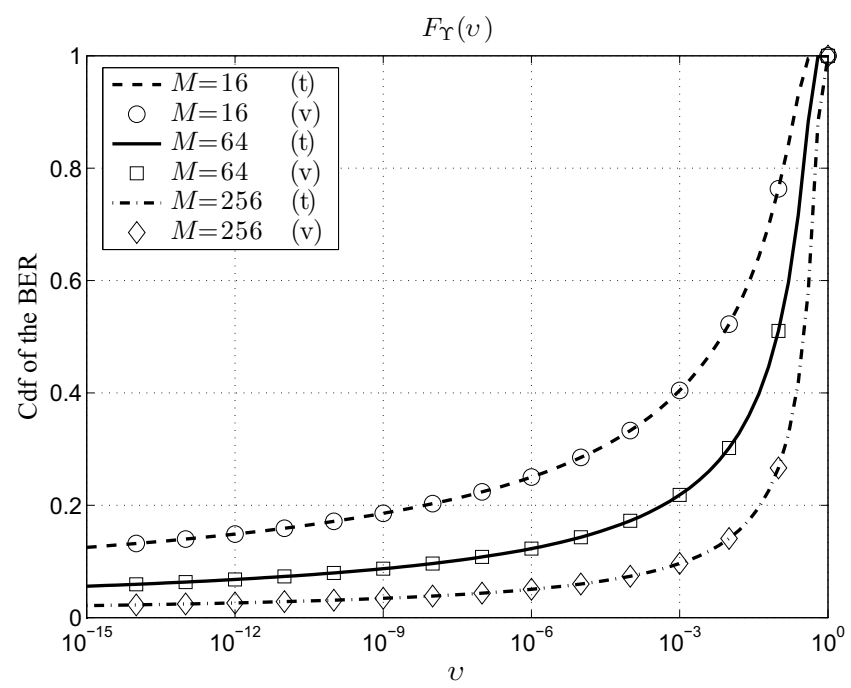

(b)

Fig. 3. Cdf of the BER for $\varrho=100 \mathrm{~m}$ and different values of the modulation order: (a) $M$-PSK, (b) $M$-QAM (t: theory, v: Monte Carlo validation).

however, the derived results may be considered satisfactory, since they have been obtained with conservative assumptions regarding the selected parameters. In fact, larger transmission powers, until $1 \mathrm{~W}$, and higher antenna gains, until $20 \mathrm{~dB}$, are usually chosen in the available experimental setups [21].

\section{Conclusions}

A theoretical analysis for calculating the statistics of the received signal power and of the BER for a $5 \mathrm{G}$ mmWave uplink communication in LOS conditions has been presented. Closed-form expressions have been derived for the cdf of the BER when the $M$-PSK and $M$-QAM schemes are used in the presence of Poisson-distributed users. The developed model has enabled to investigate the impact of the cell radius and of the modulation order on the performance of the MT-BS uplink. Ongoing research efforts are devoted to the extension of the model to non-LOS scenarios and to the deepening of the aspects related to user mobility, with particular reference to those determined by mid- and small-scale fading.

\section{REFERENCES}

[1] J.G. Andrews, S. Buzzi, W. Choi, S.V. Hanly, A. Lozano, A.C.K. Soong, and J.C. Zhang, "What will 5G be?" IEEE Journal on Selected Areas in Communications, vol. 32, no. 6, pp. 1065-1082, June 2014.

[2] R. Corrado, M. Comisso, and F. Babich, "On the impact of the video quality assessment in $802.11 \mathrm{e}$ ad-hoc networks using adaptive retransmissions," in IEEE IFIP Annual Mediterranean Ad Hoc Networking Workshop (Med-Hoc-Net), 2-4 June 2014, pp. 47-54.

[3] A. Seetharaman, N. Niranjan, V. Tandon, S. Devarajan, M.K. Moorthy, and A.S. Saravanan, "What do customers crave in mobile 5G? A survey spotlights four standout factors," IEEE Consumer Electronics Magazine, vol. 6, no. 3, pp. 52-66, July 2017.

[4] G.A. Akpakwu, B.J. Silva, G.P. Hancke, and A.M. Abu-Mahfouz, "A survey on 5G networks for the Internet of Things: Communication technologies and challenges," IEEE Access, vol. 6, pp. 3619-3647, Feb. 2018.

[5] F. Babich, M. Comisso, A. Crismani, and A. Dorni, "On the design of MAC protocols for multi-packet communication in IEEE 802.11 heterogeneous networks using adaptive antenna arrays," IEEE Transactions on Mobile Computing, vol. 14, no. 11, pp. 2332-2348, Nov. 2015.
[6] D. Neirynck, C. Williams, A. Nix, and M. Beach, "What do customers crave in mobile 5G? A survey spotlights four standout factors," IET Microwaves, Antennas and Propagation, vol. 1, no. 6, pp. 1170-1176, Dec. 2007.

[7] F. Babich, M. Comisso, and A. Dorni, "A novel SIR-based access scheme for multi-packet communication in 802.11 networks," in IEEE International Conference on Communications (ICC), 10-15 June 2012 pp. 1-5.

[8] F. Babich and M. Comisso, "Including the angular domain in the analysis of finite multi-packet peer-to-peer networks with uniformly distributed sources," IEEE Transactions on Communications, vol. 64, no. 6, pp. 2494-2510, June 2016

[9] S. Singh, R. Mudumbai, and U. Madhow, "Interference analysis for highly directional $60-\mathrm{GHz}$ mesh networks: The case for rethinking medium access control," IEEE/ACM Transactions on Networking, vol. 19, no. 5, pp. 1513-1527, Oct. 2011.

[10] T. Bai and R.W. Heath Jr., "Coverage and rate analysis for millimeterwave cellular networks," IEEE Transactions on Wireless Communications, vol. 14, no. 2, pp. 1100-1114, Feb. 2015.

[11] M. Di Renzo, "Stochastic geometry modeling and analysis of multitier millimeter wave cellular networks," IEEE Transactions on Wireless Communications, vol. 14, no. 9, pp. 5038-5057, Sep. 2015.

[12] H. Shokri-Ghadikolaei, C. Fischione, G. Fodor, P. Popovski, and M. Zorzi, "Millimeter wave cellular networks: A MAC layer perspective," IEEE Transactions on Communications, vol. 63, no. 10, pp. 3437-3458, Oct. 2015.

[13] V.M. Nguyen and M. Kountouris, "Performance limits of network densification," IEEE Journal on Selected Areas in Communications, vol. 35, no. 6, pp. 1294-1308, June 2017.

[14] M. Cheng, J.-B. Wang, Y. Wu, X.-G. Xia, K.-K. Wong, and M. Lin, "Coverage analysis for millimeter wave cellular networks with imperfect beam alignment," IEEE Transactions on Vehicular Technology, vol. 67 no. 9, pp. 8302-8314, Sep. 2018.

[15] M. Comisso and F. Babich, "Coverage analysis for 2D/3D millimeter wave peer-to-peer networks," IEEE Transactions on Wireless Communications, vol. 18, no. 7, pp. 3613-3627, July 2019.

[16] A. Boscolo, F. Vatta, F. Armani, E. Viviani, and D. Salvalaggio, "Physical AWGN channel emulator for Bit Error Rate test of digital baseband communication," Applied Mechanics and Materials, vol. 241244, pp. 2491-2495, Dec. 2013.

[17] P.C. Pinto and M.Z. Win, "Communication in a Poisson field of interferers - Part I: Interference distribution and error probability," IEEE Transactions on Wireless Communications, vol. 9, no. 7, pp. 2176-2186, July 2010.

[18] P.C. Pinto and M.Z. Win "Communication in a Poisson field of interferers - Part II: Channel capacity and interference spectrum," IEEE Transactions on Wireless Communications, vol. 9, no. 7, pp. 2187-2195, July 2010. 
[19] F. Vatta, A. Soranzo, and F. Babich, "Low complexity bound on irregular LDPC belief-propagation decoding thresholds using a Gaussian approximation," Electronics Letters, vol. 54, no. 17, pp. 1038-1040, Aug. 2018.

[20] F. Babich and M. Comisso, "Impact of segmentation and capture on slotted Aloha systems exploiting interference cancellation," IEEE Transactions on Vehicular Technology, vol. 68, no. 3, pp. 2878-2892, Mar. 2019.

[21] M.R. Akdeniz, Y. Liu, M.K. Samimi, S. Sun, S. Rangan, T.S. Rappaport, and E. Erkip, "Millimeter wave channel modeling and cellular capacity evaluation," IEEE Journal on Selected Areas in Communications, vol. 32, no. 6, pp. 1164-1179, June 2014

[22] F. Babich, M. Comisso, and A. Cuttin, "Impact of interference spatial distribution on line-of-sight millimeter-wave communications," in VDE European Wireless (EW), 16-19 May 2017, pp. 363-368.

[23] F. Babich and M. Comisso, "Multi-packet communication in heterogeneous wireless networks adopting spatial reuse: Capture analysis," IEEE Transactions on Wireless Communications, vol. 12, no. 10, pp. 53465359, Oct. 2013.

[24] L.I. Vaskelainen, "Iterative least-squares synthesis methods for conformal array antennas with optimized polarization and frequency properties," IEEE Transactions on Antennas and Propagation, vol. 45, no. 7, pp. 1179-1185, July 1997.

[25] M. Comisso, G. Buttazzoni, and R. Vescovo, "Reconfigurable antenna arrays with multiple requirements: A versatile 3D approach," International Journal of Antennas and Propagation, vol. 2017, pp. 1-9, Article ID6752108.

[26] B. Fuchs and J.J. Fuchs, "Optimal polarization synthesis of arbitrary arrays with focused power pattern," IEEE Transactions on Antennas and Propagation, vol. 59, no. 12, pp. 4512-4519, Dec. 2011.

[27] R.M. Corless, G.H. Gonnet, D.E.G. Hare, D.J. Jeffrey, and D.E. Knuth, "On the Lambert W function," Advances in Computational Mathematics, vol. 5, no. 1, pp. 329-359, Dec. 1996.

[28] R.D. Yates and D.J. Goodman, Probability and stochastic processes. New York, John Wiley and Sons, 1999.

[29] J. Lu, K.B. Letaief, J.C-I Chuang, and M.L. Liou, "M-PSK and M-QAM BER computation using signal-space concepts," IEEE Transactions on Communications, vol. 47, no. 2, pp. 181-1849, Feb. 1999.

[30] J.G. Proakis and M. Salehi, Digital Communications. New York, McGraw-Hill Higher Education, 2008.

[31] H. Shokri-Ghadikolaei and C. Fischione, "The transitional behavior of interference in millimeter wave networks and its impact on medium access control," IEEE Transactions on Communications, vol. 64, no. 2 , pp. 723-740, Feb. 2016. 